\title{
Diagnosis and treatment of lymphocutaneous dermatosis caused by Nocardia brasiliensis: a case report
}

\author{
Yan Chen^, Ying Liu, Xiao-Jie Ding, Yu-Juan Chen, Li Wang, Zheng-Zhong Zhang \\ Department of Dermatology, Affiliated Hospital of North Sichuan Medical College, Nanchong, China \\ Correspondence to: Dr. Zheng-Zhong Zhang. Department of Dermatology, Affiliated Hospital of North Sichuan Medical College, Nanchong, China. \\ Email: 3603766574@qq.com.
}

\begin{abstract}
Cutaneous nocardiosis is a skin disease mainly caused by Nocardia brasiliensis and Nocardia asteroides. Here, we report a rare case of lymphocutaneous dermatosis in an 87-year-old Chinese man infected with Nocardia brasiliensis. An 87-year-old Chinese man presented at our hospital after suffering erythema, nodules, abscesses, ulceration, and pain in the left upper limb for 10 days. The patient was initially misdiagnosed as lymphocutaneous sporotrichosis. The results of gram staining, acid-fast staining, mass spectrograph revealed Nocardia brasiliensis and 16S ribosomal RNA (16S rRNA) sequencing of samples showed that the patient had a Nocardia brasiliensis infection. Anti-infective therapy with sulfamethoxazole combined with amoxicillin clavulanate potassium was administered for 10 days, followed by sulfamethoxazole alone for 20 days. After 30 days of treatment, the abscess was treated with repeated pus extraction, debridement of erosion and ulcer, wet compress of povidone iodine solution and spectrum of multi-source instrument. The redness and swelling had subsided, and purulent secretion and ulceration had decreased. Lymphocutaneous nocardiosis can easily be misdiagnosed as sporotrichosis based on its clinical manifestations. However, mass spectrometry analysis showed Nocardia brasiliensis according to the fingerprint of the bacteria and $16 \mathrm{~S}$ rRNA sequencing to identify bacterial DNA can assist with making a diagnosis. For patients with Nocardia brasiliensis, sulfamethoxazole combined with amoxicillin clavulanate potassium is an effective anti-infective treatment.
\end{abstract}

Keywords: Lymphocutaneous nocardiosis; Nocardia brasiliensis; diagnosis; treatment

Submitted May 09, 2020. Accepted for publication Aug 21, 2020.

doi: 10.21037/apm-20-1301

View this article at: http://dx.doi.org/10.21037/apm-20-1301

\section{Background}

Primary cutaneous nocardiosis caused by Nocardia brasiliensis is an uncommon disease in clinical practice (1). The clinical and radiological characteristics of the disease mean that misdiagnosis is common, which often leads to the condition becoming aggravated, or even death (2-4). Here, we report a case of lymphocutaneous dermatosis in an 87-year-old Chinese man infected with Nocardia brasiliensis. We present the following case in accordance with the CARE reporting checklist (available at http://dx.doi.org/10.21037/ apm-20-1301).

\section{Case presentation}

\section{Chief complaints}

An 87-year-old male patient presented at our hospital with a 10-day history of erythema and papules on his left arm with no obvious cause. Then the papules burst and erosion appeared (Figure 1A). Oral cephalosporin treatment administered in a local clinic did not improve the patient's condition. The skin damage extended to the forearm and cubital fossa. The patient developed erythema and nodules, gradually forming abscesses, which ruptured causing

'ORCID: Yan Chen, https://orcid.org/0000-0002-5908-3192. 


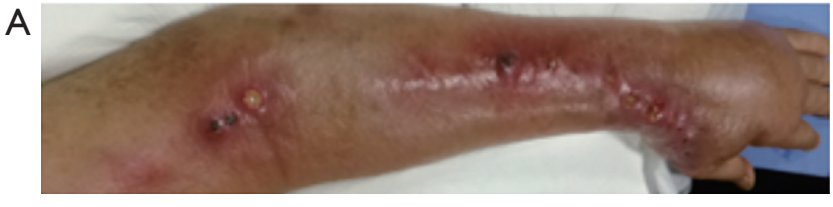

B

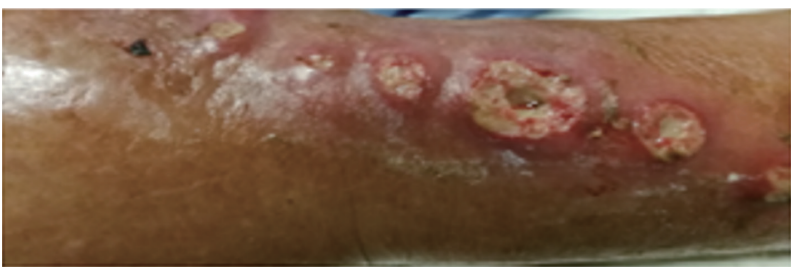

Figure 1 Skin lesions infected with Nocardia brasiliensis and the treatment response. (A) Skin lesions on the left forearm, upper arm, and left thumb; (B) some skin lesions had ruptured, producing purulent secretions and ulceration.

pain. The patient had a 7-year history of coronary heart disease, with long-term use of oral cardiac medication, and active asthma symptoms. A diagnosis of lymphocutaneous sporotrichosis was considered according to the characteristic skin lesions.

\section{History of past illness}

The patient had a 7-year history of coronary heart disease, for which he had been taking long-term oral cardiac medication.

\section{Family bistory}

The patient had no history of hypertension or diabetes, and no similar disease in the family.

\section{Physical examination upon admission}

Physical examination of the patient in the dermatology department of our hospital showed there to be extensive erythema and papules with rupture and purulent secretion on the left arm. Additionally, edema and erythema with nodules and abscesses of various sizes were observed on the left forearm and elbow. The tubercle was hard and had partially ruptured at the top, with secretions and ulceration. The lesions were distributed as beads along the lymphatic vessels, and the back of the left hand was observed to be swollen (Figure 1). The patient was initially diagnosed with lymphocutaneous sporotrichosis.

\section{Laboratory examinations and imaging examinations}

The results of laboratory tests revealed that the patient's liver and kidney function, $\mathrm{T}$ cell immunity, tumor markers, and routine stool and urine tests were normal. Computed tomography (CT) and ultrasonography of the head and chest found no obvious abnormalities. Routine blood tests showed a neutrophil ratio of $86.6 \%$ (normal range, $50-70 \%$ ). Ultrasonic examination of the skin and soft tissue showed edema, nodular thickening, and fissured effusion from the left forearm to the carpal dorsum . Color Doppler echocardiography showed widening of the aortic sinus and ascending aorta and decreased left ventricular diastolic function. The secretion was negative for fungal culture, and culture of surface purulent secretion showed white gypsum powder-like colony growth (Figure $2 A$ ). The results of hematoxylin and eosin (H\&E) staining and improved acidfast staining were positive (Figure 2B,C). Mass spectrograph revealed Nocardia brasiliensis. Gene identification by $16 \mathrm{~S}$ ribosomal RNA (16S rRNA) sequencing showed Nocardia brasiliensis (Figure 2D).

\section{Final diagnosis}

Based on the above tests and examination results, the patient's final diagnosis was lymphocutaneous nocardiosis.

\section{Treatment and outcome}

Anti-infective therapy with compound trimethoprimsulfamethoxazole (TMP-SMX, $0.96 \mathrm{~g}, 3$ times/day) combined with amoxicillin clavulanate potassium $(1.2 \mathrm{~g}$, 3 times/day) was administered for 10 days, followed by sulfamethoxazole alone, debridement of abscesses, and external application of normal saline on the purulent areas for 20 days. After 30 days' treatment, the abscess was treated with repeated pus extraction, debridement of erosion and ulcer, wet compress of povidone iodine solution and spectrum of multi-source instrument. The rash on the patient's left arm had become dry and scabby. The scabs subsequently fell off, the swelling in the left elbow subsided, purulent secretion decreased, and the rash mostly subsided (Figure 3).

All procedures performed in studies involving human participants were in accordance with the ethical standards of the institutional and/or national research committee(s) and with the Helsinki Declaration (as revised in 2013). Written informed consent was obtained from the patient. 

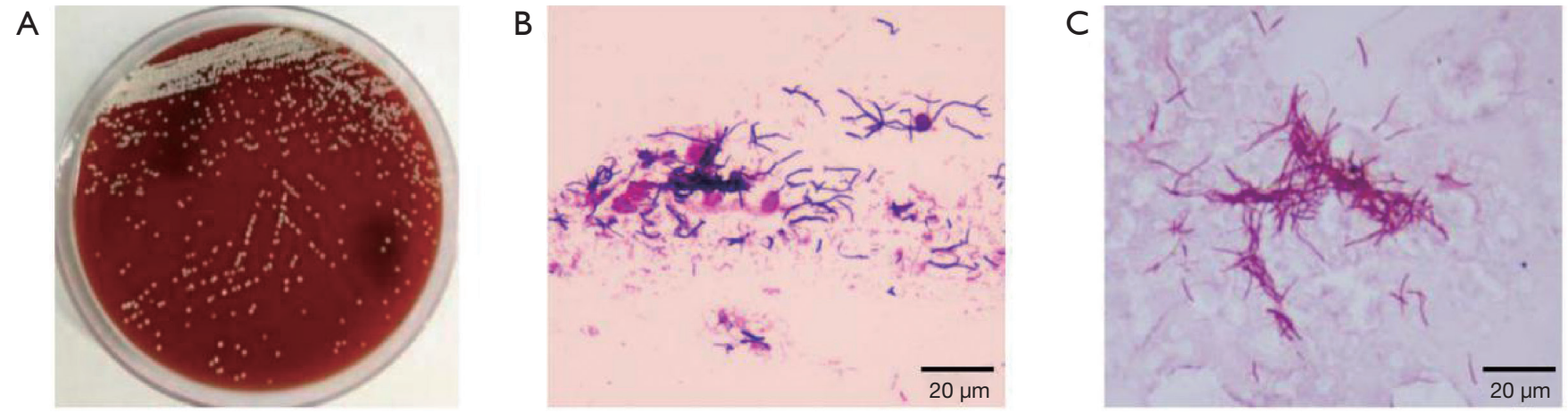

$\mathrm{D}$

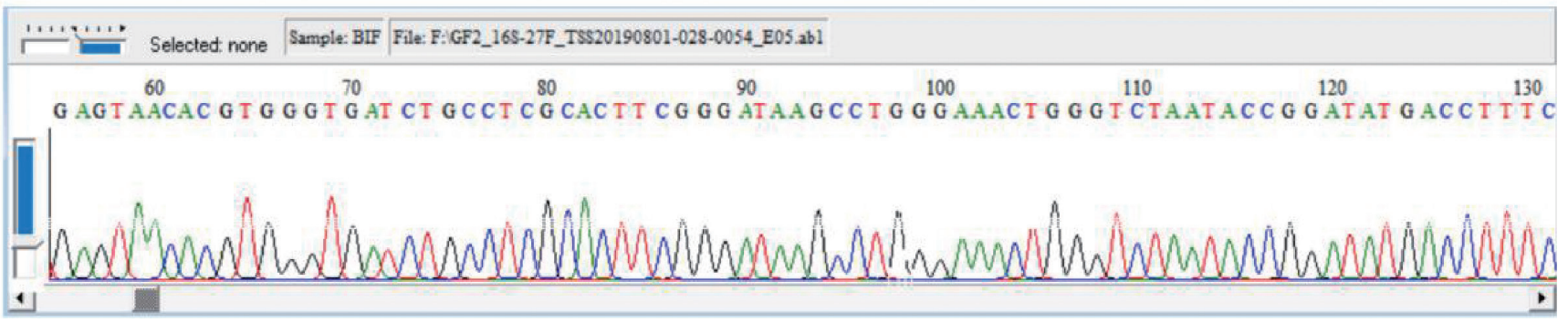

Figure 2 Bacterial culture, staining, and gene sequencing. (A) White bacterial colonies on Sabouraud agar medium. (B) Gram staining showed a large number of blue hyphae. (C) Acid-fast staining showed red rod mycelium. (D) Sequence peak of 16S rRNA gene of Nocardia brasiliensis.

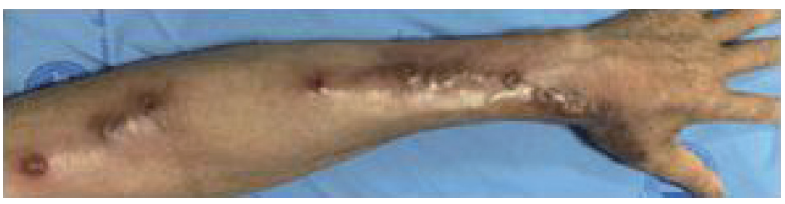

Figure 3 After 4 weeks of treatment, the skin lesions were dry, scabby, and without concentrated secretion.

\section{Discussion}

Nocardia is an opportunistic pathogen commonly found in decaying animals and plants, soil, dust, and air, which can cause lung, skin, central nervous system, and disseminated infections. It is an aerobic actinomycete with Grampositive staining of filamentous areas and weakly positive acid-fast staining. Common Nocardia species include Brazil Nocardia, Star Nocardia, Africa Nocardia, Anthrax Nocardia, and abscess Nocardia $(1,2)$. While nocardiosis often occurs in individuals who have low immune function, it can also affect individuals with normal immune function. Nocardia primarily infects the body following inhalation, which mainly causes pulmonary infection that can easily be misdiagnosed as pulmonary tuberculosis or pulmonary tumor (5). Some Nocardia infections result in skin ulceration, or hematogenous dissemination. Nocardiosis cutis, which is mainly caused by infection with Nocardia, can be divided into primary and secondary infection (primary superficial nocardiosis and lymphocutaneous nocardiosis ) (4,6). Lymphocutaneous nocardiosis initially presents as ulcerative plaques at the site of inoculation, before the infection diffuses through the lymphatics, causing lymphangitis and subcutaneous erythematous nodules, which upon rupturing may discharge pus and sulfur-like particles (7).

The primary diagnosis of lymphocutaneous nocardiosis is based predominantly on local skin plaques and ulcers, lymphangitis resulting from rapid spread along the lymphatics, as well as subcutaneous erythema, nodules, ulcers, and other clinical manifestations. The primary diagnosis is mainly informed by the results of Gram staining and acid-fast staining (positive), mass spectrograph revealed Nocardia brasiliensis. 16S rRNA sequencing usually 
forms the basis for accurate diagnosis. Lymphocutaneous nocardiosis can easily be misdiagnosed as Cutaneous lymphosporidiosis, which usually occurs after a wound is exposed to sporotrichosis due to minor trauma and is mainly manifested as chronic infection of the skin, subcutaneous tissue, and nearby lymph nodes, as well as erythema, nodules, and ulceration. Misdiagnosis of lymphocutaneous nocardiosis as atypical molecular bacilli, erysipelas, cellulitis, erythema nodosum, or another disease is also possible.

Because of variations in the pathogenicity and antibiotic sensitivity of different Nocardia species, there is currently no unified standard treatment for Nocardial infection. Sulfonamides (including trimethoprim and sulfamethoxazole) are considered to have the greatest effect in the treatment of Nocardia infections. However, there are a number of reports of patients showing resistance to these drugs $(8,9)$. Imipenem, ceftriaxone, linezolid, levofloxacin, amikacin, amoxicillin clavulanate potassium, clarithromycin, and cefotaxime, are some of the drugs that can be used in place of or in combination with sulfonamides (10), and can reduce the drug resistance of xanthamines. Clinical treatment depends on the patient's drug sensitivity and the severity of their condition.

In the case described here, we treated the infection using the combination of sulfamethoxazole and amoxicillin clavulanate potassium. Initially, the patient was treated with anti-infective therapy for 2 weeks in the form of compound sulfamethoxazole (TMP/SMX; $0.96 \mathrm{~g}, 3$ times/day) combined with amoxicillin clavulanate potassium $(1.2 \mathrm{~g}$, 3 times/day). This was followed by sulfamethoxazole treatment for another 2 weeks, as well as abscess debridement and external application of normal saline to the purulent areas. After 4 weeks of treatment, the ulceration and purulent secretion had decreased, and the rash, redness, and swelling had subsided.

\section{Conclusions}

This case illustrated that lymphocutaneous nocardiosis can be misdiagnosed as sporotrichosis based on its clinical manifestations. Nocardiosis should be considered when skin nodules and ulcers that show no response to conventional treatment are observed. To assist with diagnosis, mass spectrograph revealed Nocardia brasiliensis, 16S rRNA sequencing to identify bacterial DNA can be performed. For nocardiosis caused by Nocardia brasiliensis, sulfamethoxazole combined with amoxicillin clavulanate potassium is an effective anti-infective therapy.

\section{Acknowledgments}

Funding: None.

\section{Footnote}

Reporting Checklist: The authors have completed the CARE reporting checklist. Available at http://dx.doi.org/10.21037/ apm-20-1301

Conflicts of Interest: All authors have completed the ICMJE uniform disclosure form (available at http://dx.doi. org/10.21037/apm-20-1301). The authors have no conflicts of interest to declare.

Etbical Statement: The authors are accountable for all aspects of the work in ensuring that questions related to the accuracy or integrity of any part of the work are appropriately investigated and resolved. All procedures performed in studies involving human participants were in accordance with the ethical standards of the institutional and/or national research committee(s) and with the Helsinki Declaration (as revised in 2013). Written informed consent was obtained from the patient.

Open Access Statement: This is an Open Access article distributed in accordance with the Creative Commons Attribution-NonCommercial-NoDerivs 4.0 International License (CC BY-NC-ND 4.0), which permits the noncommercial replication and distribution of the article with the strict proviso that no changes or edits are made and the original work is properly cited (including links to both the formal publication through the relevant DOI and the license). See: https://creativecommons.org/licenses/by-nc-nd/4.0/.

\section{References}

1. Chen B, Tang J, Lu Z, et al. Primary Cutaneous Nocardiosis in a Patient With Nephrotic Syndrome. Medicine 2016;95:e2490.

2. Baio PVP, Ramos JN, Santos LSD, et al. Molecular Identification of Nocardia Isolates from Clinical Samples and an Overview of Human Nocardiosis in Brazil. PLoS Neglected Tropical Diseases 2013;7:1-10.

3. Nosotti M, Tarsia P, Morlacchi LC. Infections after lung transplantation. J Thorac Dis 2018;10:3849-68.

4. Lee SH, Sung H, Lee SO, et al. The first report of disseminated Nocardia concave infection in an immuno- 
compromised patient in South Korea. J Infect Chemother 2012;18:764-6.

5. Majeed A, Abdullah HM, Ullah W, et al. First reported case of disseminated Nocardia kroppenstedtii sp nov. infection presenting with brain abscess and endocarditis in an immunocompromised patient with mantle cell lymphoma: challenges in diagnosis and treatment. BMJ Case Reports 2017;2017:bcr2016217337.

6. Gilquin JM, Riviere B, Jurado V, et al. First case of actinomycetoma in France due to a novel nocardia species, nocardia boironii sp nov. Msphere 2016;1:e00309-16.

7. Banerjee B, Rohit G, Muralidhar V, et al. Disseminated Nocardia asiatica infection in an immunocompromised individual: A rare entity needs careful vigilance. J Infect

Cite this article as: Chen Y, Liu Y, Ding XJ, Chen YJ, Wang L, Zhang ZZ. Diagnosis and treatment of lymphocutaneous dermatosis caused by Nocardia brasiliensis: a case report. Ann Palliat Med 2020;9(5):3663-3667. doi: 10.21037/apm-20-1301
Public Health 2019;12:167-70.

8. Gazzoni FF, Severo LC, Marchiori E. Pulmonary Diseases with Imaging Findings Mimicking Aspergilloma. Lung 2014;192:347-57.

9. Boamah H, Puranam P, Sandre RM. Disseminated Nocardia farcinica in an immunocompetent patient. IDCases 2016;6:9-12.

10. Chaudhari DM, Renjen PN, Sardana R, et al. Nocardia Farcinica Brain Abscess in an Immunocompetent Old Patient: A Case Report and Review of Literature. Ann Indian Acad Neurol 2017;20:399-402.

(English Language Editor: J. Reynolds) 